\title{
Application Analysis of Blockchain Technology in the Field of Emergency Management
}

\author{
Wang Qiquan, Shao Wenpeng \\ Department of Safety Engineering, China University of Labor Relations, Beijing, China \\ Email address: \\ wqq_100@163.com (Wang Qiquan),576484586@qq.com (Shao Wenpeng)
}

\section{To cite this article:}

Wang Qiquan, Shao Wenpeng. Application Analysis of Blockchain Technology in the Field of Emergency Management. Advances in Applied Sciences. Vol. 5, No. 2, 2020, pp. 49-56. doi: 10.11648/j.aas.20200502.15

Received: February 9, 2020; Accepted: February 25, 2020; Published: June 17, 2020

\begin{abstract}
Aiming at the current situation of emergency management in China, this paper discusses the research status of emergency management construction in China, the main problems existing, and the basis for establishing an emergency management system. Briefly introduce the concept and technical characteristics of the blockchain, propose the irreversible, distributed, and difficult to tamper characteristics of the block-chain technology, and apply the advantages that can be brought about in emergency management. The management of equipment and facilities in the enterprise emergency management system is the starting point, the establishment of special equipment to run life-cycle block chain technology with the entire process is analyzed, and then the system is associated with big data, to make it more intelligent and reasonable. In the end, it is concluded that the application of blockchain technology to the entire life cycle of management equipment and facilities can achieve excellent results. Based on the combination of blockchain technology and big data, it can be applied to all stages of emergency management to achieve security. Information management is the inevitable trend of future emergency management. Although the construction of the emergency management system is a complex project, the construction is difficult, the cycle is long, and many issues are involved, but with the emergence of new technologies, the emergency management system is bound to be constantly improved and taken to a whole new level.
\end{abstract}

Keywords: Emergency Management, Blockchain, Big Data, Enterprise, System Construction

\section{Introduction}

At the 46th World Economic Forum Davos Annual Meeting, blockchain was included in the "Fourth Industrial Revolution" along with artificial intelligence and autonomous driving. With the launch of the bitcoin block chain technology has become the Internet field, especially in the financial technology sector fiery shall concept, attracting more and more attention, research, and even investment. 2019 Nian 10 Yue 24-day President Xi Jinping in the eighteenth Politburo collective study also stressed the importance of breakthrough should block chain as the core technology innovation, focus on breakthroughs in core technologies to expedite the implementation of the block Chain technology and industrial innovation and development.

Block chain technology has already begun applied in many industries, but particularly in the field of emergency management is the application of emergency management in the enterprise is very small, emergency management as a core Public Security management, in 2018 Nian 3 months, especially the thirteen Responsibilities of each department were integrated to form the Emergency Management Department. Since the establishment of the Emergency Management Department, China's emergency management situation has generally improved. But more of them are in the improvement of government functional departments, and although the company has greatly improved the overall safety management system, its entire emergency management process is far from the government's management. In recent years, major disasters have also occurred frequently. Since 2013, there have been a number of extremely serious accidents in China, such as Jilin Baoyuan, Tianjin Binhai New Area, Shenghua, Hebei, and Xiangshui, Jiangsu. Very bad effect. The occurrence of the above accidents revealed that most of the accident companies' equipment and facilities have been in disrepair for many years, and there is no record of relevant information. It has gone through several hands, several inspections and repairs, how to inspect and repair, 
whether the parts are solid, whether the quality is qualified, and the containers. There is no way to know the hazards of internal substances and other information. If the equipment fails and the relevant information is not understood, it will not only affect the emergency response measures of rescuers, but also the judgment of the cause of the accident. Sometimes emergency rescue is not only ineffective, it may even cause the accident and disaster to worsen. However, if these data are stored in the blockchain, the cryptographic principles and hard-to-tamper characteristics of the blockchain technology can not only protect the authenticity and accuracy of the data, but also provide accurate information when the equipment is in a state of failure. Make emergency rescue more rational. The scientific application of new technologies to improve the emergency management of enterprises is of great significance for promoting safe development and establishing a long-term mechanism for safe production.

This article starts with the current situation and development of emergency management in China, and describes the core technical characteristics of the blockchain and the foundation of the emergency management system based on the blockchain technology, emergency management related laws and regulations, and computer program language. It also takes the control and prevention of equipment and facilities as the starting point in the establishment of an emergency management system, and builds the entire life cycle of government supervision and enterprise operation of special equipment under blockchain technology. In order to realize efficient and sensitive government information management, rapid response, reliable operation of safety information management, provide a preliminary idea of a safety information platform, improve the level of production safety supervision, monitoring and accident emergency for enterprises, and provide an information-based approach.

\section{Overview of China's Emergency Management Research}

\subsection{Current Situation of Emergency Management Research in China}

China started research on emergency management of late, for a long period of time, whether government or academia, emergency management were not given enough attention, from the outbreak of the 2003 "SARS" epidemic, social development and public Life has caused serious damage, and the importance of emergency management has gradually been realized by scholars, and then the theoretical research related to emergency management has really developed.

From 2003 to 2007, domestic research on emergency management entered a development period. XueLan and others put forward the concept of establishing a crisis management system, which pointed out the direction for the research of emergency management system in China's academic circles. The main research result at this stage was the emergency management system based on "one case, three systems". Initial formation. However, most of the research on emergency management in this period stayed at the national macro level, and the research on emergency management from the micro level was relatively scarce $[1,2]$.

From 2008 to 2018, domestic research on emergency management reached a boom period. The emergency management system based on the "one case, three systems" framework was deepened and strengthened. Xie Yuanqing and Zhou Hui followed closely the development trend of the times, researched on the background of the era of big data, analyzed in depth the problems existing in China's emergency management system, and provided innovative ideas for the theoretical research of emergency management [3].

In March 2018, in order to prevent and resolve heavy safety risks, improve the public safety system, integrate and optimize emergency forces and resources, the responsibilities of the State Administration of Work Safety and the General Administration of State Council emergency management responsibilities were integrated. Set up the emergency management department.

Since the establishment of the Emergency Management Department, it is obvious to all that China's emergency management has improved significantly. However, in recent years, accidents and disasters have occurred frequently in enterprises, and many problems have been exposed, including: insufficient understanding of emergency management. Regional and departmental leaders did not have a high understanding of emergency management of public emergencies, did not attract enough attention, and were not active enough in their work. The company's awareness of safety in production is weak, and it is reluctant to increase capital investment in safety in production. Equipment and facilities have been out of repair for a long time, and the number of protective equipment to be equipped is seriously inadequate; emergency management agencies are not sound. In some places, there is no emergency management agency, especially in the counties, townships, and villages. The emergency management work is very weak, or even in a laissez-faire state. Once an emergency occurs, it will inevitably delay the best time for rescue; the emergency plan is targeted, the operability is not strong. Although regions, their departments, and enterprises have formulated emergency plans for emergencies, due to insufficient research and inadequate argumentation, the classification standards for plans are not scientific, reasonable, and accurate. The prepared emergency plans will inevitably have poor predictability, poor operability, and classification. Response and emergency command regulations are not specific, and coordination and coordination between departments are inadequate; related science and technology and application gaps are large. Computers, modern communications, satellites, remote sensing, information, and biotechnology are far from being applied to emergency management and emergency rescue work. The emergency rescue team has low-level technical 
equipment and equipment, lacks advanced rescue equipment with high technological content and strong practical performance, and emergency management and emergency rescue staff who have mastered high and new technology. Therefore, various sophisticated technologies are strengthened in the emergency management of enterprises. Application is very meaningful.

\subsection{The Foundation of Establishment of Enterprise Emergency Management System}

The establishment of an emergency management system is a large and complex systematic project that involves before, during, and after an accident. Before the accident, enterprises should do a good job of accident prevention and preparation, including the implementation of the safety responsibility system, the improvement of rules and regulations, the preparation and exercise of emergency plans, the training of safety education, the management of equipment and facilities, the management of the operating environment, etc. If an accident occurs, the enterprise should immediately carry out emergency response work, rescue personnel, control dangerous sources, and evacuate the people. After the accident, the leaders and management personnel must take the lead to carry out on-site cleanup and recovery, find out the cause of the accident, and find out the recovery of the accident responsibility. Therefore, its establishment needs the necessary support from many aspects.

\subsubsection{Emphasis on the Person in Charge of the Enterprise and the Management}

To establish an emergency management system, a lot of basic work must be done, and a considerable amount of human and material resources must be invested, which requires sufficient attention and support from the person in charge and management of the enterprise. The person in charge of the company mainly controls the overall situation, implements the company's production safety responsibility system, rules and regulations, and operating procedures, ensures the effective input of safety funds, and organizes the preparation of the unit's emergency rescue plan. Management personnel must participate in the work of the person in charge of the enterprise and provide support. They must also organize their own production safety education and training, implement management measures for major sources of danger, check the status of production safety at the unit, and promptly investigate the hidden dangers of production safety accidents. To stop and correct violations of command and operation rules; make clear that emergency management of the enterprise is an inevitable direction for future work, to enable employees to establish a security concept of "preventing risks, removing hidden dangers, and curbing accidents" and actively participate in the construction of emergency management systems among.

\subsubsection{Talents with Professional and Technical strength}

The construction of the emergency management system is a systematic project that involves a wide range of professional areas and strong technical requirements. On the one hand, it needs the support of professionals in production safety science and management, because the times are developing, various new technologies are emerging, and management and technical personnel more and more knowledge is needed, but the management of most companies is generally older at the present time. Under the current technology, it is obvious that the company should not perform security management and emergency management. Most of the management depends on many years of experience. The introduction of professional talents, the management methods for different technologies and different personnel also vary from person to person, and often achieve the effect of doing more with less; on the other hand, computer science, communication technology and sufficient technical support capabilities are required to provide long-term System maintenance [4], big data technology has realized secure information management. Its intelligence and scientific can allow us to provide early warning before an accident, effective response during an accident, and accurate analysis after an accident.

\subsubsection{Guarantee of Equipment, Facilities and Materials}

There are many equipment and facilities involved in the entire emergency management system, and sufficient funding is required. Such as computer purchase costs, new system design, development, maintenance costs; various equipment and facilities maintenance, maintenance, and timely replacement costs; labor protection equipment provision costs; company production safety education and training costs and fire extinguishers, fire equipment, etc. Cost of emergency relief supplies.

\section{Overview of Blockchain Technology}

Bitcoin has developed rapidly in recent years and has become the most influential virtual currency in the global market. It can be said, block chain technology is available with a bit of money, but with the deepening of research on the block chain, and now, block chain technology has been widely applied in the financial sector, such as insurance payments. Is also available in the real economy of supply chain management, product traceability, copy right in. Virtually all industries need a trustworthy trading environment as a prerequisite for development. Blockchain creates trust through mathematical principles rather than third-party intermediaries, which can more securely transmit value on the network and create more supply and demand $[5]$.

\subsection{What is a Blockchain: a Trusted Distributed Database}

Blockchain technology is, in a narrow sense, a distributed database that connects blocks in chronological order and ensures that they cannot be tampered with and forged in a cryptographic manner. It can also be called distributed ledger technology. Under normal circumstances, the system will set the data transaction records in the block to be replaced and 
broadcasted within each time period. During this time period, all the system's data information and transaction records will be placed in a newly generated block through the Markel tree data structure. If all user nodes that have received the broadcast agree with the transaction records and data information in this block, this block will be added by each user node to its original chain in a chain-like form (consensus mechanism).

As shown in the figure, the structure of the blockchain and the core technologies applied in the structure are included. The block can be roughly divided into two parts: a block header and a block body. The block header generally includes the previous block hash value (parent hash), Merkel root, timestamp, difficulty, and other information. A block body with verified, all the blocks of data records occurring in the creation process, by Merkel tree (the Merkel Tree stored) data structure, and then to ensure that each data block is encrypted by asymmetric cryptography embodiment safety. All data is recorded in the "leaf" node of this tree, and it cannot be tampered with. It traces back from level to level, and finally comes down to a tree root, otherwise it goes back to each transaction details through the tree root [6].

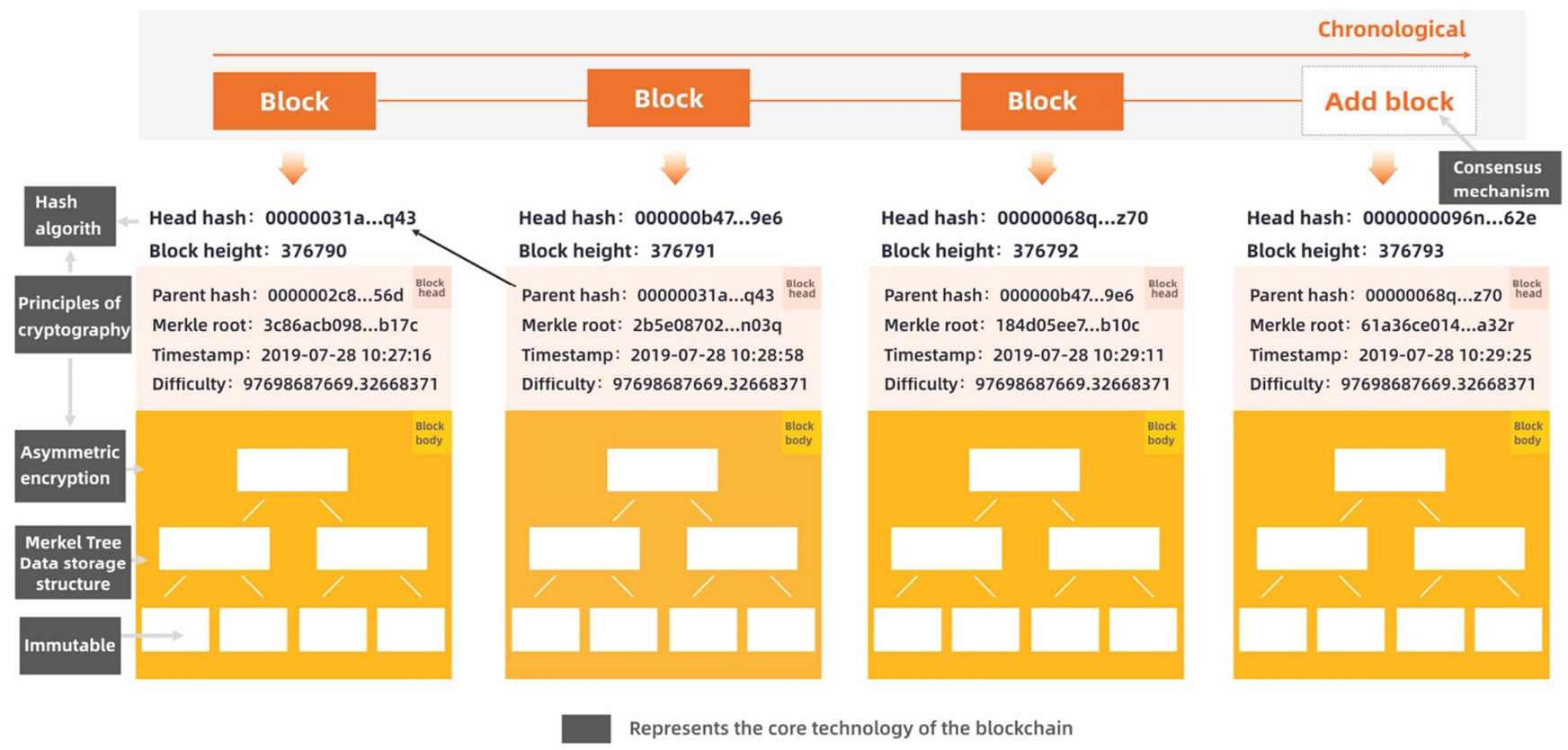

Figure 1. Blockchain architecture and core technology.

\subsection{Blockchain Core Technology Introduction}

\subsubsection{Distributed Accounting and Storage}

Distributed means that the data in the blockchain is recorded (accounted) and stored by all user nodes in the system, and each participating user node has a complete blockchain information, even if there are some nodes

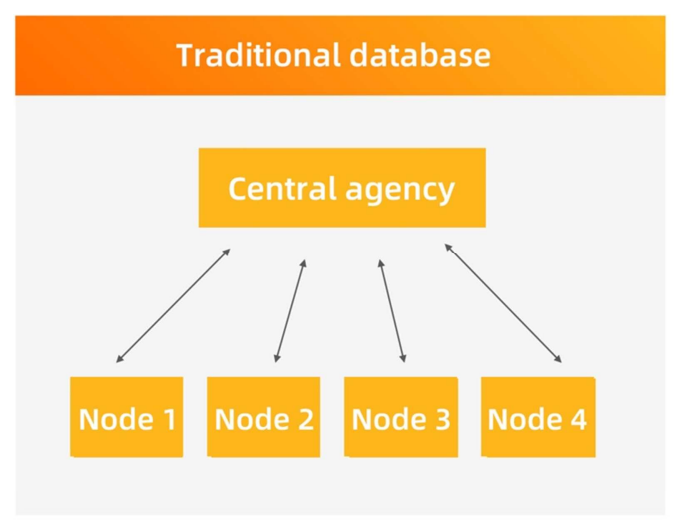

Information is tampered with or changed by an attack, and it will not cause any impact on the entire network. These user nodes do not need to trust or recognize each other, nor do they need to belong to the same organization. This is not possible with traditional databases.

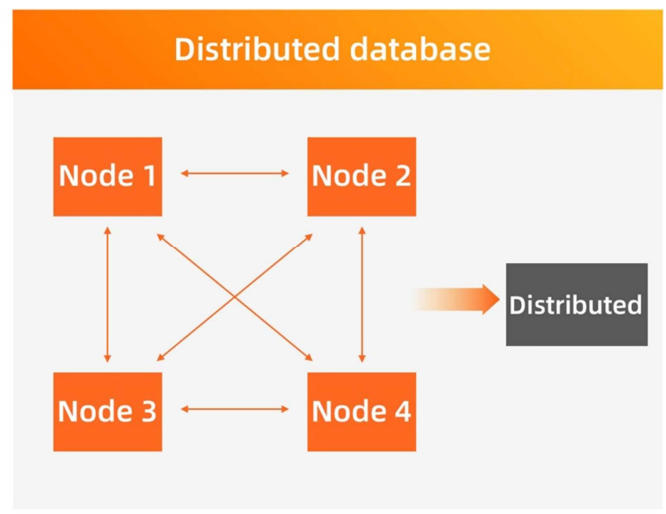

Represents the core technology of the blockchain

Figure 2. Distributed database. 


\subsubsection{Cryptographic Hash Algorithm}

Hash algorithm is an encryption algorithm, which is a very important technology in the field of information. Entering arbitrary information characters can produce a fixed-size hash output. In layman's terms, we can understand the output of the hash algorithm as the "identity number" of each user in the blockchain world. Just like in the real world, each of us has his own unique ID and ID number, we can also use a specific and unique hash value to represent a block, just like we can't fall from the "ID number" Like pushing out your name, age, gender and other related information, you can't infer the specific content of the block from the hash value, which reflects the privacy of the hash function.

\subsubsection{Data Storage Structure-Merkel Tree}

The Merkel Tree is actually a data structure. It is used to summarize all transactions in a block. The root of the tree is the hash value of the entire transaction set. It is calculated from the hash values of all data blocks below the root of the tree, as long as any data in the tree is tampered with the hash of the root node will not match, which can achieve verification purposes.

\subsubsection{Consensus Mechanism}

In simple terms, the mechanism by which blockchain nodes reach a consensus of user nodes across the network on transaction data information can be referred to as a consensus mechanism, which can ensure that the latest blocks are accurately and trusty added to the blockchain and can even resist the actions of criminals attack on purpose.

\subsubsection{Immutable}

If you want to tamper with information in the blockchain, it is very difficult. The high security of data is the best embodiment of immutable technology. This is the main difference between blockchain data and traditional databases.

As shown in the figure 3 , if we want to tamper with a piece of data in the blockchain, the head hash of the current block will change, and the changed head hash will not match the parent hash of the next block. Tampers need to continue to modify the parent hash of the next block, and continue to modify each subsequent block. This requires that tampers simultaneously invade all participating nodes and tamper with the data at the same time, which is difficult to happen in real life.

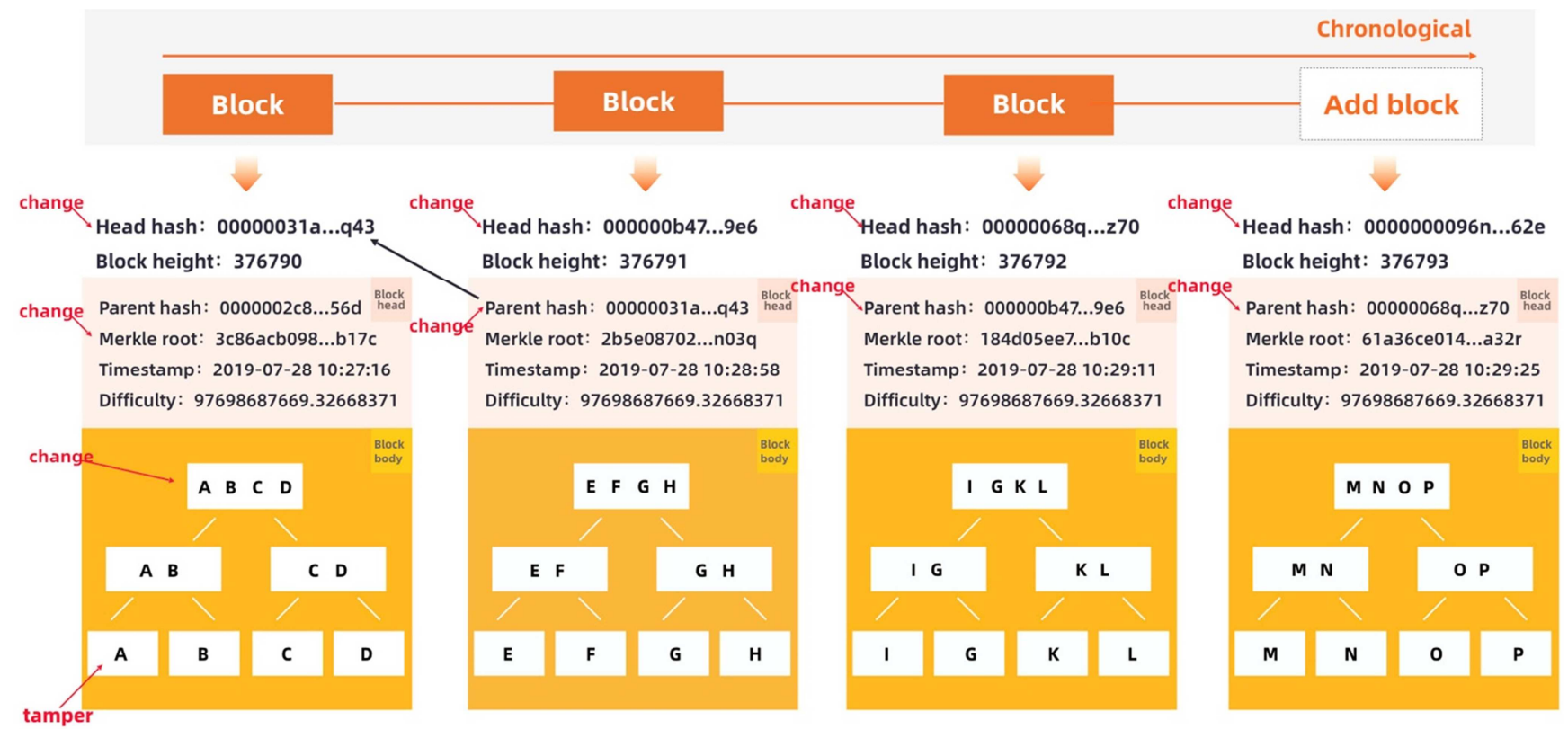

Figure 3. Immutable.

\section{Application of Blockchain in the Field of Emergency Management}

At present, blockchain technology has begun to be applied in various industries, but it can be said that there are very few applications in the emergency field. The blockchain is a chronologically traceable, secure and reliable data blockchain. It has the core technical advantages of being irreversible and difficult to tamper with. Therefore, it is necessary to use the technical advantages of the blockchain to improve the efficiency of the four phases of emergency management.

\subsection{Block Chain in the Enterprise Emergency Construction Management System in Should Be Used}

In the construction of the emergency management system, the protection of enterprise equipment and facilities is an indispensable part, it is the core force to improve the economic efficiency of the enterprise, and it is also the part that the company needs to prevent. It involves the implementation of the responsibilities of company leaders and managers, the implementation of professional capabilities of professional and technical personnel and how to provide reasonable funding for equipment and facilities. 
Special equipment as a high-risk devices, often leads to serious accidents, and there will be aging equipment in most of the cause of the accident, fell into disrepair, but this was scrapped and all the missing information using over-age and so on, so play The ability of professional and technical personnel to apply blockchain technology to special equipment of enterprises, and establish the entire life cycle operation of special equipment with blockchain technology as the core for application analysis, as shown in the figure 4 .

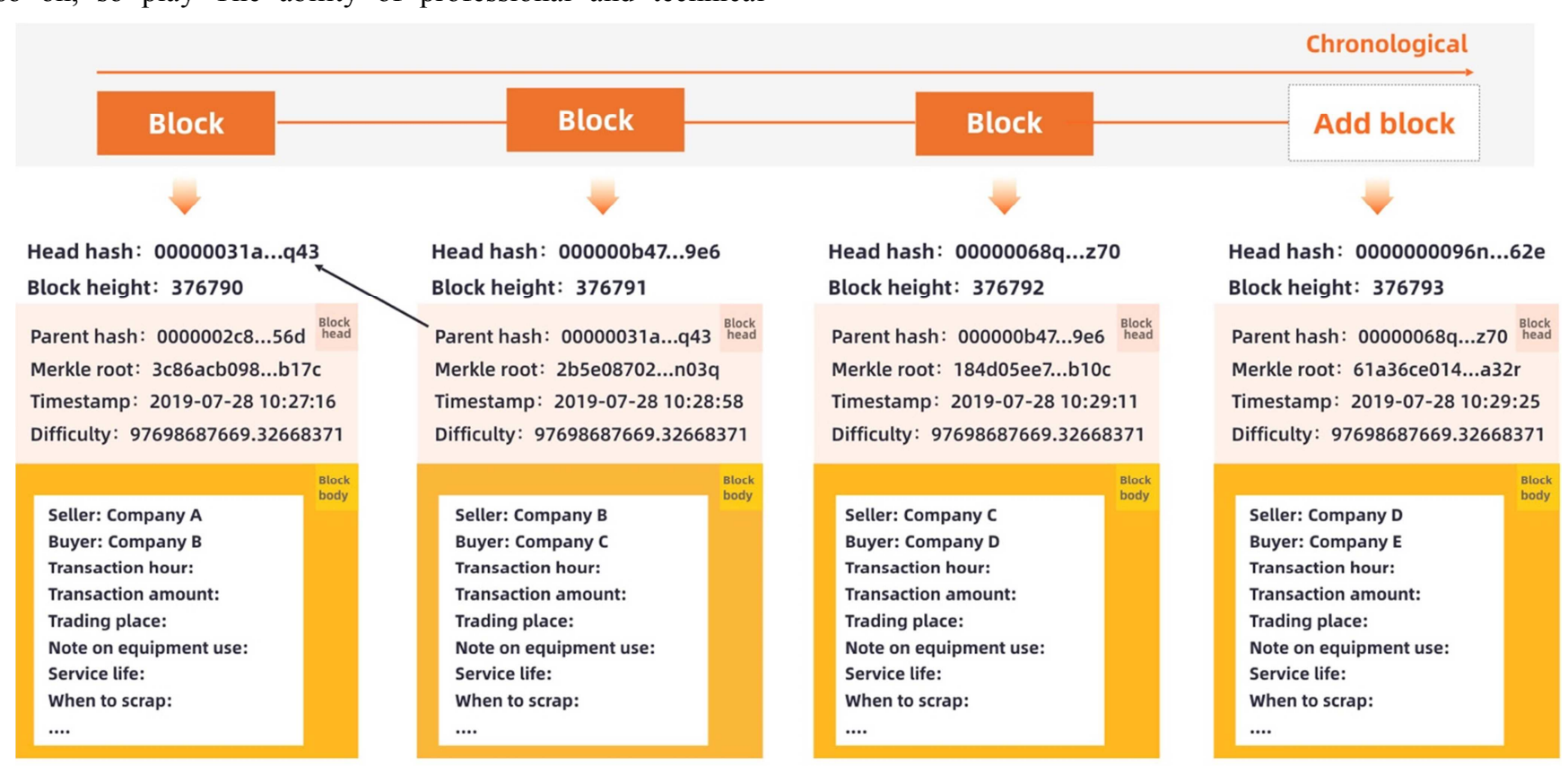

Construction of production equipment life cycle based on blockchain technology

Figure 4. Life cycle of special equipment based on blockchain technology

Establish the entire process of the life cycle of special equipment with blockchain technology as its core, its ex-factory date, each transaction information, which unit it is currently in, the time of regular inspection, the date of scrap, whether it has reached the scrap requirement, and its use Attentions, etc. All the processes related to the operation of the device, as long as it is placed in the data storage structure of the block body, all the questions can be determined and the only answer. This is beneficial to the person in charge and management of the enterprise to know the relevant information accurately, and to ensure the effective implementation of special equipment and facilities funding. Meanwhile, since the block chains are arranged in chronological order, which makes each of a data in only one direction of the display cannot reverse modification, tampering, which is the block chain security has been respected where people. After establishing the life cycle of the special equipment, the enterprise can effectively manage and control the special equipment during the preventive preparation phase of the emergency management of the special equipment. During the response phase, the authenticity of the accident information can be guaranteed, and emergency personnel can make the most reasonable as for the countermeasures, during the recovery phase, since the blockchain is a traceable and secure data chain, it is clear at a glance who checks and inspects the equipment, which is conducive to accident investigation and personnel disposal. Therefore, the application of blockchain in equipment and facilities not only promotes the establishment of the emergency management system, but also improves the four-stage application of enterprise emergency management.

After the entire life cycle of the special equipment established by blockchain technology is run, the traceability and authenticity of a lot of information can be guaranteed, but whether there are problems with the equipment have been repaired, whether the enterprise will replace them after the retirement age, etc. It is uncertain whether the problem will be solved well. Therefore, on the basis of establishing the entire process of the life cycle operation of the special equipment of blockchain technology, a government and enterprise information reporting platform associated with big data is established, as shown in the figure. It is shown that by linking big data technology to the data in the blockchain, after entering relevant information about special equipment in the big data platform, it can automatically identify the date of regular inspection and comprehensive inspection of each special equipment and provide real-time notification in advance. It can also automatically identify the information such as the date of manufacture of the equipment, and in each transaction report the real-time status of the special equipment, the useful life, the remaining useful life, precautions, etc. to the real-time notification of the latest transaction of the company's company leaders, managers and government. It is also possible to transfer the government's Industry connection point to point, when there are equipment needs inspection, maintenance, registration matters, the big 
data platform notify designated personnel and government enterprises designated person in charge of the whole process of docking matter and recorded in the new block, ensure traceability, real After the occurrence of special equipment accidents, the responsibilities of government personnel, the responsibilities of enterprise docking personnel, and the daily management duties of special equipment can be traced back to facilitate accident investigation. This will greatly improve the responsible attitude of relevant personnel and ensure the quality of each security issue

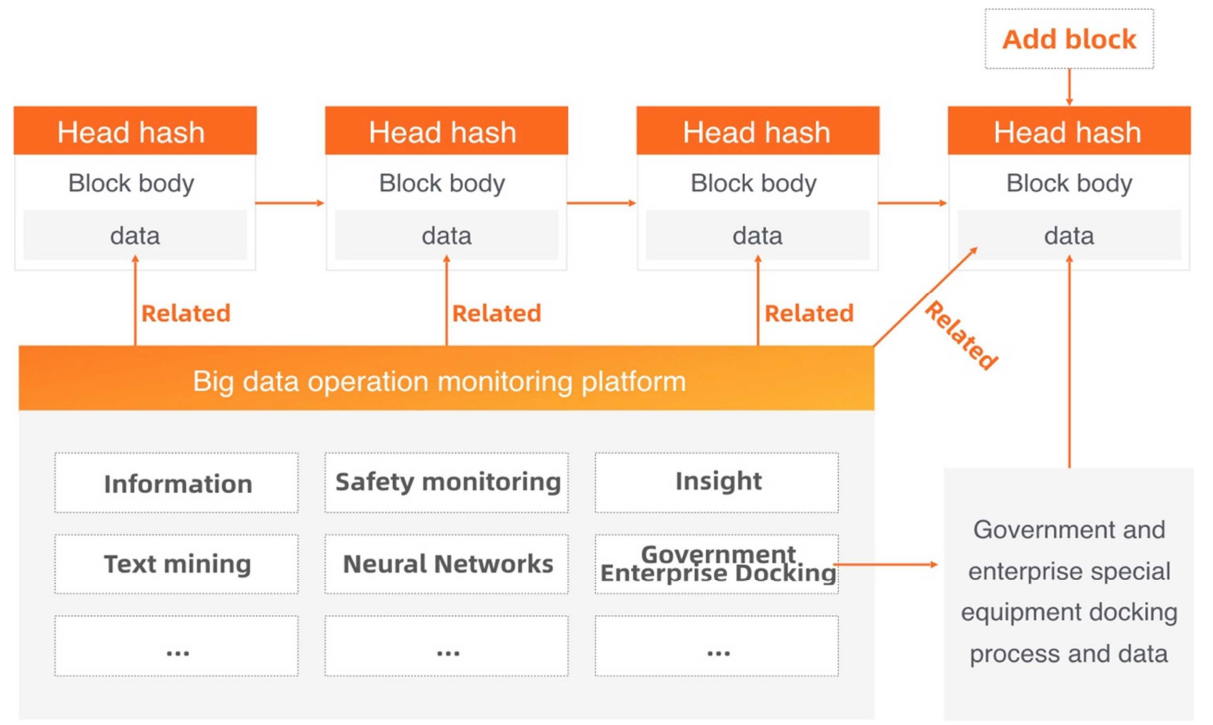

Figure 5. Government and enterprises control special equipment of enterprises based on "big data + blockchain".

\subsection{Problems Existing in the Application of Blockchain Technology in the Emergency Field}

(1) Network resources and storage consumption is too large, on how many nodes have a number of times stored, and particularly large amount of data, the time a new node is added also becomes long, the application of emergency management data resources throughout a process, which has great demands on the strength of the enterprise itself, if the enterprise storage volume overload, is likely to pose a risk Caton even system crashes.

(2) The cost is large and the suitable scenarios are still relatively limited. Compared with traditional equipment and facilities, the advantages of blockchain technology are that it is highly efficient, robust, and highly reliable in data recording by virtue of decentralization. However, many of the traditional security manufacturers in funding showed insurmountable defect and fault aspects. So compared to the cost of renovation to be paid, a business if evolution is a block chain technology systems can bring benefits than traditional systems how can more than how much, or just the data you want to save or build a platform to use, whether significant cost Being able to get back better economic benefits is the key issue that most enterprises consider when investing in emergency management.

(3) Laws, regulations, and policies are not complete. At present, there is no universal standard for blockchain at home and abroad. The subsequent applications have compatibility and interoperability problems, which is not conducive to the improvement of overall efficiency.

\section{Conclusion}

(1) With the popularization of blockchain technology, it will inevitably be more and more widely used in all stages and stages of the enterprise emergency management field. Interpersonal communication, or transactions between business organizations, honesty and transparency are the cornerstones of mutual trust between two or more parties. Blockchain's "honesty" and "transparency" also make it highly expected to become the "trust machine" of the Internet. The application of blockchain technology to emergency management to achieve information management of security management is also an inevitable trend of future emergency management.

(2) With the rapid development of science and technology and socialized large-scale production, the safety of production information management based on big data is becoming increasingly important in emergency management. Achieving the effective integration of enterprise safety production information management and enterprise management will be an advanced model of enterprise information development by leaps and bounds. Enterprise safety information is an indispensable resource for management activities. Safety management information system is an indispensable "neural system" in the entire emergency management system.

(3) Improving the enterprise emergency management system is a complex system project. It is based on a 
large amount of work. It is a system that involves many departments and has diverse requirements. The construction is difficult and the cycle is long. Although blockchain technology and big data technology can only solve some of these problems, with the emergence of new technologies, it is bound to continue to improve the construction of the emergency management system and take it to a whole new level.

\section{Acknowledgements}

This paper is supported by general project of China University of Labor Relations (20XYJS017).

\section{References}

[1] Xu Wenhui, Zhang Chengfu. Government Management in Crisis [M]. Renmin University of China Press, 1998.

[2] Xue Lan, Zhang Qiang, Zhong Kaibin. Crisis Management: Challenges Facing China in the Transition Period [J]. China Soft Science, 2003 (04).

[3] Xie Yuanqing, Zhou Hui. China's emergency management system innovation in the era of big data [J]. China Management Informationization, 2016 (04).

[4] Wang Qiquan. Research on Construction of Enterprise Safety Production Information Management System [J]. Chinese Journal of Safety Science, 2010, 20 (5): 109-113.

[5] Lian Yixi. Blockchain research report: How far is it from the trust machine to the industrial wave [J]. Development Research, 2018 (8): 16-29.
[6] Zhou Minwen. Tan Haiwen. Analysis on the status quo and countermeasures of information safety construction in China [J]. Surface Mining Technology, 2005 (6): $36 \sim 38$.

[7] Li Lihui. Interpreting the Blockchain [J]. China Finance, 2019, (22): 25-27.

[8] Blockchain accelerates industrial integration [J]. Software and Integrated Circuits, 2019 (11): 20.

[9] Xiao Feng. Blockchain is a machine of trust [J]. Shanghai Branch Life, 2019, (12): 36-37.

[10] Blockchain: A New Engine for Industry Innovation [J]. Software and Integrated Circuits, 2019 (11): 18-19.

[11] Yang Dong. Blockchain needs to be regulated [J]. China Real Estate Finance, 2018 (12): 78.

[12] Yang Yiqun. Autonomous Driving Technology Based on Big Data and Blockchain [J]. Time Automobile, 2019 (15): 35.

[13] Zhang Honglei, Su Ying. Research on the Development of Sharing Economy Based on "Big Data + Blockchain" Technology [J]. The Think Tank Times, 2019 (37): 17-18.

[14] Jiang Yantong, He Chenchen, Zhang Jiayi et al. Discussion on the development of "Big Data + Blockchain" Sharing Economy [J]. Zhifu Times, 2019 (3): 51.

[15] Xiong Ling, Tang Weipeng, Li Zhongliang. Research on Blockchain Application of Big Data Platform in University Library $[\mathrm{J}]$. Science and Technology Literature Information Management, 2019, 33 (3): 49-52.

[16] Ding Dandan, Hu Bingqian, Jiang Fan et al. Development direction of blockchain in the financial field in the era of big data [J]. Heilongjiang Science, 2019, 10 (11): 154-156. 